\title{
Transition to process model parameters radical polymerization
}

\author{
(C) Marina N. Patrusheva, ${ }^{1+}$ Eleanor R. Mukhamedzyanova, ${ }^{2}$ and Rafail M. Khuzakhanov ${ }^{1 *}$ \\ ${ }^{1}$ Department of Chemistry and Technology of Processing Elastomers; ${ }^{2}$ Scientific and Technological \\ Center of Chemistry and Petrochemistry. Kazan National Research Technological University. \\ K. Marx St., 68. Kazan, 420015. Republic of Tatarstan.Russia.E-mail: ${ }^{1)}$ marina.patrol91@mail.ru
}

Keywords: radical polymerization, mathematical model, gel effect, styrene.

*Supervising author; ${ }^{+}$Corresponding author

\begin{abstract}
A mathematical transition to model parameters is presented, which are decisive in the preparation of a mathematical model of the radical polymerization process taking into account the gel effect. Using the mathematical model of radical thermal polymerization of styrene as an example, the derivation of parameters is considered, taking into account which the values of the number-average and mass-average molecular weights obtained as a result of calculation using similar parameters given in the previously published literature are confirmed. The difference of the study is the description of a step-by-step transition to the model parameters, applicable to the description of the radical polymerization process of other monomers, taking into account the gel effect.
\end{abstract}

\section{References}

[1] J.A. Beisenberger, D.H. Sebastian. Engineering problems of polymer synthesis. Moscow: Chemistry. 1988. P.202-203. (russian)

[2] A.W. Hui, A.E. Hamielec. Thermal polymerization of styrene at high conversions and temperatures. An experimental study. J. Appl. Polym. Sci. 1972. Vol.16. No.3. P.749-769.

[3] N.V. Ulitin, K.A. Tereshchenko. Methods for modeling the kinetics of synthesis processes and the molecular weight characteristics of polymers. Kazan: Publishing House of KNITU. 2014. P.22. 51. (russian)

[4] W.E. Gibbs, K.R. Fisch, M. Gehatia, I.J. Goldfarb. Polymerization kinetics by means of differential thermal analysis. ML Technical Documentary Report. 1964. P.64-136. 